\title{
The Research on Game Theory of Resources for Free Based on Personal Beliefs
}

\author{
Wei Zhang \\ College of Science \\ Wuhan University of Science and Technology \\ Wuhan, 430065, China
}

\author{
Shuifang Yin \\ College of Science \\ Wuhan University of Science and Technology \\ Wuhan, 430065, China
}

\begin{abstract}
Problems of resources for free often be associated with personal interests. Personal beliefs make people choose to get resources differ in time,so-called first come first served rule. So people access to resources constitute a game under the conflict of interests. In this paper, we study two men's personal beliefs under taking the optimal strategies firstly,we use the influences of participation time to amount of resources acquired to get the optimal resource acquisition strategies for two men and secondly, we use the expected utility functions to obtain their expected utility of resources, finally we base on the personal beliefs to explore their belief functions under the optimal strategies are adopted, further we gain relative true faiths, that provides certain theoretical basis for the game of resources strategies.
\end{abstract}

Keywords-resources acquisition; game theory; strategies; expected utility function; belief functions

\section{INTRODUCTION}

Resources for free means resources need not be exchanged through the material and get it,the quantity of resource to gain only relate to personal beliefs. Resources for free seemed to be simple,but it closely related to personal beliefs to a large extent,for the size of personal beliefs will influences those who gain resources on taking the strategies,that is early start and late start to gain resources will produce different results.So,it makes a game among those who gain resources,thus thereis a close relationship between the size of the personal beliefs and how many resources they could obtain.

In this paper, we study two persons' free access to resources,and suppose that there is no difference among all resources'use to people, meanwhile assume that the amount of all resources is 1.Two persons aimed to obtain the corresponding resources to maximize their own interests,and assume that there is no excessive gain.At the same time,we suppose that resources for free only relate to personal beliefs,all correlative factors of obtaining resources are reflected in the personal beliefs for choices. Personal beliefs directly reflect in two persons when select early start or late start to gain resources,therefore,the time of early start or late start comes to be a bridge between personal beliefs and the resource acquisition.

Firstly consider the impact of the chioce of time on the resource acquisition,then quantify the two men's optimal strategies,finally use relative real belief functions to obtain personal beliefs under taking the optimal strategy. For effects on the time sequence,the time two men spend to arrived in the place of resources may be different,that is to say choose early start or late start will be the only condition for two men to make a decision.On the other hand,due to the assume time sequence depend on personal beliefs, and resources the two men obtained rely on their time sequence,thus beliefs become the only factor which determines the resource access.

\section{GAME THEORY OF RESOURCES FOR FREE}

Mark two men as $A$ and $B$,and the resources makes their interests to maximize are $m$ and $n$ respectively,meanwhile $0<m, n<1$.For $m$ and $n$,following two cases.

\section{A. Obtaining Resources without Conflict}

If $m+n \leq 1$,then two men could obtain resources they want no matter what choices they make,that means whoever firstly start to obtain resources can maximize their own interests.For who arrived in place of resources early the other reached lately could obtain resources he want,so two men are able to get what they need to maximize their own interests.

\section{B. Obtaining Resources with Conflict}

When $m+n>1$, the resources they need is a kind of situation in the conflict,that is to say,all the resources two men need will be more than 1 . Now research what influences the time sequence will produce in this case that two men obtain resources under conflict.

(1) $A$ access to resources early than $B$.Then the resource

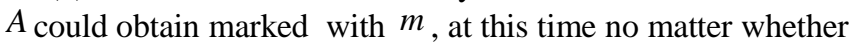
$B$ will compete with $A, A$ is able to maximize his own interests,and $B$ could gain resources of $1-m$.

(2) $B$ access to resources early than $A$.Then the resource $B$ could obtain marked with $n$, at this time no matter whether $A$ will compete with $B, B$ is able to maximize his own

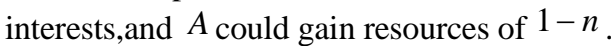

(3) $A$ and $B$ start to the place of resources at the same time.There exists competition between them.Indeed,two men obtain resources under conflict,so they need a fair compromise according to rational under this situation,only do this could they obtain a certain amount of resources.Now give a percentage,that they access to the resources according to the percentage to wreck. Thus both of them should in accordance with the percentage for a fair compromise. 
Mark the percentage as $p$,and $p=\frac{m+n-1}{m+n}$,it represent the exceed resources two men needed beyond the total resources. When two men all start early to gain resources, due to the resources they need is more than 1 , so everyone should give up a certain proportion of resources to avoid the happening of the conflict between two men. At this time the quantity of resource $A$ gains is $m-m \cdot p=m(1-p)=\frac{m}{m+n}$, and the quantity of resource $B$ gains is $n-n \cdot p=n(1-p)=\frac{n}{m+n}$. When two men all start lately to gain resources, the resources they need is more than 1 too, also under this situation, the resources should has some losses according to the rational for two men need more time to arrive at the place of resources,this part of losses embodied in the decrease of the amount of resource acquisition. Stipulate the resources they gain on the basis of the concession have part of wreck, under this situation the quantity of resource $A_{\text {could gain is }} \frac{m}{m+n} \cdot \frac{1-n}{m}=\frac{1-n}{m+n}$ and the quantity of resource $B$ could gain is $\frac{n}{m+n} \cdot \frac{1-m}{n}=\frac{1-m}{m+n}$.

As we have mark the resources $A$ and $B$ need to gain as $m$ and $n$, the following form is the game form about early start and late start to arrive at the place of resources.

$B$

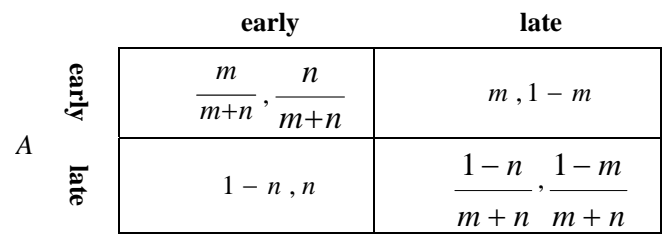

FIGURE I. GAME FORM

From the form we can conclude that different strategies influent the quantity of resource they could obtain, and the choice of strategies made by two men depend on themselves, two men plan the strategies of their decisions with the size of the probability.

\section{QUANTITATIVE ANALYSIS OF THE Optimal STRATEGY}

Two men's optimal strategies which can maximize their own interests could be shown by expected utility function. Expected utility function theory established the framework under the condition of uncertainty to analysis the rational men's choose, it is a way of analysis used to deal with uncertainty decision problems[1]. For two people take different strategies to obtain resources, we can use the expected utility function to calculate their optimal resource they could gain.

Use the expected utility function to seek the two relative optimal strategies of the game, two theorems are given respectively by the following.
Theorem 1 In the game of two men's resource acquisition, A choose the probability of $\theta$ to start early, and probability of $1-\theta$ to start lately, the mixed strategy of $A$ is $(\theta, 1-\theta)$,then, if $A$ choose the probability of $\theta^{*}=\frac{1-n}{1-n+m}$ to start early he could maximize his interests.

Proof As the mixed strategy of $A$ is $(\theta, 1-\theta)$,so the expected utility function of $A$ is as follows:

$$
u_{A}=\theta\left[\frac{m}{m+n} \cdot \gamma+m(1-\gamma)\right]+(1-\theta)\left[(1-n) \cdot \gamma+\frac{1-n}{m+n}(1-\gamma)\right]
$$

Computing the differential of $u_{A}$, and get a first-order condition of optimization:

$$
\frac{\partial u_{A}}{\partial \gamma}=\theta\left[\frac{m}{m+n}-m\right]+(1-\theta)\left[(1-n)-\frac{1-n}{m+n}\right]=\frac{(1-m-n)[(1-n+m) \theta-1+n]}{m+n}=0
$$

Then, we can get $\theta^{*}=\frac{1-n}{1-n+m}$.

That is to say if $A_{\text {choose the probability of }} \theta^{*}=\frac{1-n}{1-n+m}$ to start early he could maximize his interests.

Theorem 2 In the game of two men's resource acquisition, $B$ choose the probability of $\gamma$ to start early, and probability of $1-\gamma$ to start lately, the mixed strategy of $B$ is $(\gamma, 1-\gamma)$, then, if $B$ choose the probability of $\gamma^{*}=\frac{1-m}{1-m+n}$ to start early he could maximize his interests.

Proof As the mixed strategy of $B$ is $(\gamma, 1-\gamma)$,so the expected utility function of $B$ is as follows:

$$
u_{B}=\gamma\left[\frac{n}{m+n} \cdot \theta+n(1-\theta)\right]+(1-\gamma)\left[(1-m) \cdot \theta+\frac{1-m}{m+n}(1-\theta)\right]
$$

Computing the differential of $u_{B}$, and get a first-order condition of optimization:

$$
\begin{aligned}
& \frac{\partial u_{B}}{\partial \theta}=\gamma\left[\frac{n}{m+n}-n\right]+(1-\gamma)\left[(1-m)-\frac{1-m}{m+n}\right]=\frac{(1-n-m)[(1-m+n) \gamma-1+m]}{m+n}=0 \\
& \text { Then, we can get } \gamma^{*}=\frac{1-m}{1-m+n} .
\end{aligned}
$$

That is to say if $B$ choose the probability of $\gamma^{*}=\frac{1-m}{1-m+n}$ to start early he could maximize his interests. 


$$
\begin{aligned}
& \text { Hence, the mixed strategy } \\
& A_{\text {is }}(\theta, 1-\theta)=\left(\frac{1-n}{1-n+m}, \frac{m}{1-n+m}\right) \text { and the mixed strategy of } \\
& B \text { is }(\gamma, 1-\gamma)=\left(\frac{1-m}{1-m+n}, \frac{n}{1-m+n}\right) \text {. }
\end{aligned}
$$

Above two theorems are figured out through the expected utility function of two men's different strategies to gain resources,and provide two people with relatively straightforward mixed strategy to obtain resources, as well provide a theory basis of the determination of two people belief functions.

\section{Personal Beliefs and Relative True Faith Under THE OPTIMAL STRATEGIES}

Belief functions are used to reflect the subjective judgments of the people to the problems of the objective world [2], two men's strategies are shown with belief functions, assume the Personal belief function as $b_{i}(i=A, B)$, and $F$ is a finite set. According to the two men's choices, there have early set out and late start, mark early set out as early and late start as late in brief, Through the permutation and combination we know $F=\{$ AearlyBearly, AearlyBlate, AlateBearly, AlateBlate $\}$,also call

$F$ recognition framework.

By the first section assumption, the strategy of two men's start early or late departure is only related to personal beliefs, and shown with belief functions. Then the belief function express the judgment of each other's choice, the judgment is also given in the form of probability too, of course, the belief function is the probability of a value between 0 and 1 .

The quantity of resources to be obtained is determined by both themselves and the belief functions, belief functions have different criteria for every one because of the differences between individuals. When two men obtain the same quantity of resources, we assume the size of the same quantity of resources is $t$, and $t=\alpha b_{A}, t=\beta b_{B}, \alpha, \beta \in[0,1] b_{A}, b_{B}$ represent two men's beliefs respectively, $\alpha, \beta$ represent personal fixed parameter, it means two men's executive capacities on the basis of beliefs to obtain the same quantity of resources. To avoid the belief function for two different criteria, we normalize $b_{A}, b_{B}$ and get the proportion of faith of $A$ is $\frac{t / \alpha}{\|t / \alpha+t / \beta\|}$, the proportion of faith of $B$ is $\overline{\|t / \alpha+t / \beta\|}$.

\section{A. The Relative Real Belief Function}

In the game of two men's resource acquisition, the size of the personal belief always along with change, in order to balance the reliability of the personal belief's source, define $b_{A}^{\prime}=\frac{t / \alpha}{\|t / \alpha+t / \beta\|} b_{A}+\left(\frac{t / \alpha}{\|t / \alpha+t / \beta\|}-\frac{t / \beta}{\|t / \alpha+t / \beta\|}\right) b_{B}$,

$$
b_{B}^{\prime}=\frac{t / \beta}{\|t / \alpha+t / \beta\|} b_{B}+\left(\frac{t / \beta}{\|t / \alpha+t / \beta\|}-\frac{t / \alpha}{\|t / \alpha+t / \beta\|}\right) b_{A} \text {, call }
$$
them the relative real belief functions, they are the average of personal belief function's errors, and more close to the real data of the two men's belief functions.

By the influence of two men's action to obtain resources namely resources game we can get mixed optimal strategy, which in turn can calculate the two men's expected utility of obtaining resources, further to get their own belief functions, and then to balance, get two men's relative true beliefs under the optimal strategies.

\section{B. Relative True Beliefs under the Optimal Strategies}

From a rational perspective, both $A$ and $B$ will take their optimal strategies to maximize their resource benefits, so $A$ and $B$ would start early by the probability of $\theta^{*}$ and $\gamma^{*}$ respectively. That is to say when $A$ takes the optimal strategy of start early by the probability of $\theta^{*}=\frac{1-n}{1-n+m}$ to maximize their resource benefits, meanwhile $B$ takes the optimal strategy of start early by the probability of $\gamma^{*}=\frac{1-m}{1-m+n}$

maximize their resource benefits, then $A$ and $B$ can obtain the expectations of the resource, respectively

$$
\begin{aligned}
& u_{A}^{*}=\theta^{*}\left[\frac{m}{m+n} \cdot \gamma^{*}+m\left(1-\gamma^{*}\right)\right]+\left(1-\theta^{*}\right)\left[(1-n) \cdot \gamma^{*}+\frac{1-n}{m+n}\left(1-\gamma^{*}\right)\right] \\
& =\frac{m n\left(1+m^{2}-n^{2}\right)+m\left(1-m^{2}+n^{2}\right)}{(m+n)(1-m+n)(1+m-n)} \\
& u_{B}^{*}=\gamma^{*}\left[\frac{n}{m+n} \cdot \theta^{*}+n\left(1-\theta^{*}\right)\right]+\left(1-\gamma^{*}\right)\left[(1-m) \cdot \theta^{*}+\frac{1-m}{m+n}\left(1-\theta^{*}\right)\right] \\
& =\frac{m n\left(1+n^{2}-m^{2}\right)+n\left(1-n^{2}+m^{2}\right)}{(m+n)(1-n+m)(1+n-m)}
\end{aligned}
$$

Then personal beliefs of $A$ and $B$ under maximizing their resource benefits respectively

$$
\begin{aligned}
b_{A}^{\prime} & =\frac{t / \alpha}{\|t / \alpha+t / \beta\|} b_{A}+\left(\frac{t / \alpha}{\|t / \alpha+t / \beta\|}-\frac{t / \beta}{\|t / \alpha+t / \beta\|}\right) b_{B} \\
& =\frac{t / \alpha}{\|t / \alpha+t / \beta\|} \cdot \frac{u_{A}^{*}}{\alpha}+\left(\frac{t / \alpha}{\|t / \alpha+t / \beta\|}-\frac{t / \beta}{\|t / \alpha+t / \beta\|}\right) \frac{u_{B}^{*}}{\beta} \\
b_{B}^{\prime} & =\frac{t / \beta}{\|t / \alpha+t / \beta\|} b_{B}+\left(\frac{t / \beta}{\|t / \alpha+t / \beta\|}-\frac{t / \alpha}{\|t / \alpha+t / \beta\|}\right) b_{A} \\
& =\frac{t / \beta}{\|t / \alpha+t / \beta\|} \cdot \frac{u_{B}^{*}}{\beta}+\left(\frac{t / \beta}{\|t / \alpha+t / \beta\|}-\frac{t / \alpha}{\|t / \alpha+t / \beta\|}\right) \frac{u_{A}^{*}}{\alpha}
\end{aligned}
$$


Thus, calculate the expected utility of resources under the situation of taking the optimal strategies,further get belief functions, and conclude that two men's relative true beliefs. And two men to each other to set off early or late start reflects in belief functions, $F=\{$ AearlyBearly, AearlyBlate, AlateBearly, AlateBlate $\}$ which is called recognition framework, so belief functions provide two men with certain theoretical basis to take the optimal strategies, the decisions of the four kinds of beliefs' strategies become a starting point of the game.

\section{CONCLUSIONS}

This article gains the optimal strategies and expected resource acquisition functions from the resources for free game, and then draw a conclusion about two men's real personal beliefs under optimal strategies which is based on personal belief functions, which reflects the personal belief functions and the optimal solutions, and provides a certain reliability on the personal strategies and beliefs acquisition.

\section{ACKNOWLEDGEMENT}

This paper is supported by the Natural Science Foundation of Hubei Province (2013CFA131) and Hubei Province Key Laboratory of Systems Science in Metallurgical Process (M201402).

\section{REFERENCES}

[1] ZhangYin Wei. Game theory and information economics,Shanghai people's press, 2004.

[2] Chao Fu.The research on group decision-making process based on the believe belief function,2009.

[3] Martin·j·Osborne,Ariel-rubinstein. A Course in Game Theory. China society press, 2000.

[4] Harold w. Kuhn.Classical game theory. China Renmin University Press, 2004.

[5] K.S.Chin, D.L.Xu, J.B.Yang,J.P-K Lam. Group-based ER-AHP system for product project screening. Expert Systems with Applications, 2008, 35(4):1909-1929.

[6] D.F.Li. Compromise ratio method for fuzzy multi-attribute group decision making. Applied Soft Computing,2007,7:807—817. 